Published online 2017 April 13.

Abstract

\title{
Role of FDG PET/CT Scan in Head and Neck Cancer Patients
} \author{
and Payam Mehrian ${ }^{2}$ \\ ${ }^{1}$ Masih Daneshvari Hospital, Pediatric Respiratory Diseases Research Center \\ ${ }^{2}$ Masih Daneshvari Hospital, Chronic Respiratory Diseases Research Center \\ ${ }^{3}$ Masih Daneshvari Hospital
}

Mehrdad Bakhshayesh Karam, ${ }^{1}$ Abtin Doroudinia, ${ }^{2}$ Farahnaz Aghahosseini, ${ }^{3}$ Fatemeh Kaghazchi, ${ }^{2}$

Received 2016 December 21; Accepted 2017 February 08.

\begin{abstract}
Background: PET/CT scan has emerging role in head and neck oncology with a few well-established indications, including: detection of unknown primary tumor site, tumor staging, radiotherapy planning, treatment response assessment and detection of recurrent disease. The purpose of this study is reporting PET/CT findings in head and neck cancer patients to emphasize on its role in head and neck oncology.

Methods: We reviewed our PET/CT date-base retrospectively and found 94 patients, referred with primary head and neck cancer. This is a descriptive report of PET/CT scan findings in head and neck cancer patients referred to MasihDaneshvari hospital since 2013 to 2016.

Results: The most common primary tumor sites were oral cavity (27\%) and nasopharynx (22\%). The most common indication for referral was tumor restaging (76\%) including treatment response evaluation and differentiation between recurrence and posttreatment fibrosis. In $60 \%$ of patients with negative primary tumor site, PET/CT was able to detect evidence of regional or distant metastasis. PET/CT was able to localize the primary tumor site in $66 \%$ of patients with unknown primary tumor site. We had also 19 patients with primary head and neck cancer referred for initial staging, demonstrating evidence of metastasis in $66.2 \%$ of all cases. Conclusions: Most patients are referred for restaging and demonstrate evidence of regional or distant metastasis with significant value for further treatment planning. Providing insurance coverage and familiarizing referring physicians about appropriate indications of this relatively new diagnostic modality will be to the best interest of head and neck cancer patients in long term.
\end{abstract}

Keywords: Head and Neck Cancers, PET/CT, Staging, Re-Staging

This is an abstract presented in the 33rd Iranian congress of radiology (ICR) and the 15th congress of Iranian radiographic science association (IRSA) 